\title{
Numerical Simulation of Cholera Epidemic Model with Quarantine
}

\author{
Trisilowati $^{1}$, A. Andari², M. A. Rois ${ }^{3}$, and M. H. Muzaqi ${ }^{4}$ \\ 1,2, 3,4 Department of Mathematics, Faculty of Mathematics and Sciences, Brawijaya University \\ E-mail: trisilowati@ub.ac.id ${ }^{1}$, ari_mat@ub.ac.id ${ }^{2}$,roizmuhammad.math@gmail.com ${ }^{3}$, \\ mohamadhasyimmuzaqi@gmail.com ${ }^{4}$
}

\begin{tabular}{|c|c|}
\hline A R T I CLE I N F O & ABSTRACT \\
\hline $\begin{array}{l}\text { History of the article: } \\
\text { Receive November } 11,2020 \\
\text { Revised January 1, 2021 } \\
\text { Accepted February 20, } 2021\end{array}$ & $\begin{array}{l}\text { Cholera is an acute diarrheal disease that spread quickly in an unsanitary } \\
\text { environment, and one of its control measures is employing quarantine. Therefore, } \\
\text { this research aims to construct a model for the spread of SIRQB-type } \\
\text { (susceptibles, infective, recovered, quarantine, bacteria) infectious diseases }\end{array}$ \\
\hline $\begin{array}{l}\text { Keywords: } \\
\text { Dynamic Analysis } \\
\text { Cholera } \\
\text { Epidemic Model }\end{array}$ & $\begin{array}{l}\text { through a nonlinear differential equation approach. Furthermore, the equilibrium } \\
\text { points condition and their stability were investigated using the standard dynamical } \\
\text { analysis method. The results show two points of equilibrium: the disease-free, } \\
\text { which always exists and is unstable, and the endemic, which is stable and exists }\end{array}$ \\
\hline $\begin{array}{l}\text { Correspondence: } \\
\text { Telepon: +62 81914430369 } \\
\text { E-mail: trisilowati@ub.ac.id }\end{array}$ & $\begin{array}{l}\text { results, and it shows that the rate of quarantine affects the spread of the infected } \\
\text { subpopulation. }\end{array}$ \\
\hline
\end{tabular}

\section{INTRODUCTION}

Cholera is an acute diarrheal disease caused by the Vibrio bacteria that enter the body through the food or drink consumed. Without proper treatment, an individual infected by these bacteria may die. Its transmission usually occurs due to the consumption of water contaminated with the Vibrio Cholerae bacteria. Furthermore, the infected individual in an area with a low rate of sanitation may spread the bacteria in local water sources, which result in contamination.

Some of the measures to combat the spread of this disease include treatments, vaccinations, and quarantine. These measures help to reduce the number of infected and susceptible individuals. Furthermore, the cholera vaccine given orally is an effective strategy in controlling the disease (Fung et al., 2013). On the other hand, quarantine is one way to deal with the spread by isolating and treating the infected population. Therefore, this measure is also a solution to control the number of infected individuals.

In recent years, some mathematical models related to the epidemic of cholera have been studied by some researchers, for example, (Kolaye et al., 2018; Peter et al., 2018; Tian et al., 2019; Berhe, 2020; and Che et al., 2020). Various research was conducted to determine the dynamics of its spread, including Codeco, which constructed a dynamic cholera model consisting of the susceptible and infected subpopulation. Also, the environmental components, namely bacteria subpopulations, were considered (Codeco, 2001). Next, this model was developed by Hartley et al. with the assumption that the population consists of very (hyper infectious) and less dangerous (less infectious) bacteria (Hartley et al., 2006). Berge et al. modeled a cholera epidemic with an unsaturated transmission rate, and the population was divided 
into two regions leading to migratory factors in the spread of cholera (Berge et al., 2015). In the same year, Edward and Nyerere developed the dynamics of cholera spread, assuming that the population grows logistically (Edward and Nyerere, 2015). In contrast, Lemos-Paiao, et al. developed the cholera dynamics spread with the control measures in the form of quarantine. This model assumed that the rate of bacteria change is only affected by the infected population. In addition, the constructed model assumes that the recovered population showed a rate of immune loss, therefore, may become vulnerable again (Lemos-Paiao et al., 2016). Panja proposed a model by assuming that the disease transmission rate is in the form of a bilinear and divides the bacteria population into two, including a subpopulation of bacteria in the body and the environment. Furthermore, Panja assumed that the rate of change in bacteria is only affected by the infected population (Panja, 2019). Generally, the research related to the cholera epidemic model involves bacteria growth originating from infected subpopulations (Misra et al., 2016; Sun et al., 2016; Peter et al., 2018, and Zhou, 2016). However, in reality, the Vibrio Cholerae bacteria can grow without an infected population. Therefore, the model needs to be added to the assumption that bacteria grow logistically to illustrate the dynamics of its spread.

Furthermore, the investigation by Lemos-Paiao, et al. was modified in this study by adding logistical growth to the bacteria population and ignoring the assumption that there is a degree of immune loss in the recovered subpopulation. Therefore, they are not susceptible again. Also, the dynamic analysis is carried out to determine the equilibrium point and analyze its stability. Subsequently, a numerical simulation is carried out to illustrate the result of the dynamic analysis.

\section{RESEARCH METHODS}

This research is a combination of literature review, theory, and experiment. The steps taken include:

\section{Model Construction.}

The cholera epidemic model was constructed based on previous studies' assumptions (Edward and Nyerere, 2015 and Lemos-Paiao, 2016). Five compartments were used to describe the process of transferring disease from one to the other.

2. Determine the Equilibrium Points

The equilibrium point and its existing conditions were determined by setting the right side of the differential equation to zero. Generally, there are two equilibrium points including disease-free $(I=0)$ and endemic $(I \neq 0)$.

3. Determine the Basic Reproduction Number

The basic reproduction number is determined using the next-generation matrix. A value less than one indicates that each infected individual will spread the bacteria to less than one susceptible person; therefore there is no outbreak. However, when the rate is greater than one, it indicates that each infected individual will infect more than one susceptible person therefore, an outbreak will occur.

4. Determining the Stability of the Equilibrium Points

The determination of stability begins with the linearization of the model to form the Jacobian matrix. Furthermore, it was determined based on the sign of the eigenvalues of the characteristic equation based on the Jacobian matrix at each equilibrium point formed. When all the eigenvalues of the real part are negative, then the equilibrium point is locally asymptotically stable. However, when there is at least one positive value, then it is unstable. 


\section{Numerical Simulation}

Numerical simulations are performed to illustrate the analysis results by selecting parameter values that meet the stability requirements. They were carried out using the 4th order Runge-Kutta method.

\section{RESULTS AND DISCUSSION}

\section{Model Construction}

The cholera epidemic model with quarantine was constructed based on the models of Edward and Nyerere (2015) and Lemos-Paiao (2016). Furthermore, the Lemos-Paiao model is modified by assuming that the bacteria grow logistically as in the Edward and Nyerere model (2015). Based on the results of these modifications, a new model is obtained as follows:

$$
\begin{aligned}
& \frac{d S}{d t}=\Lambda-\beta S B-\mu S \\
& \frac{d I}{d t}=\beta S B-\delta u I-\left(\alpha_{1}+\mu\right) I \\
& \frac{d Q}{d t}=\delta u I-\left(\varepsilon+\alpha_{2}+\mu\right) Q \\
& \frac{d R}{d t}=\varepsilon Q-\mu R \\
& \frac{d B}{d t}=b\left(1-\frac{B}{K}\right) B+\gamma I
\end{aligned}
$$

Where:

$S$ : Susceptible subpopulations,

I : Cholera-infected subpopulation,

$Q$ : Quarantined subpopulations,

$R$ : Recovered subpopulation,

$B$ : Bacteria population,

A: Natural birth rate,

$\mu$ : Natural death rate,

$\beta$ : The interaction rate of susceptible populations with bacteria,

$\delta:$ Quarantine rate,

$\alpha_{1}$ : Death rate due to infection,

$\alpha_{2}$ : Death rate due to quarantine,

$\varepsilon:$ The individual rate is recovered,

$K$ : Carrying capacity of bacteria population,

$u$ : Control rate,

$b:$ Bacteria population growth rate,

$\gamma:$ The bacteria growth rate which is triggered by infected subpopulation.

\section{Equilibrium Points}

The equilibrium point is obtained when $\frac{d S}{d t}=\frac{d I}{d t}=\frac{d Q}{d t}=\frac{d R}{d t}=\frac{d B}{d t}=0$. This system has two, namely the disease-free $\left(E^{0}\right)$ and endemic $\left(E^{*}\right)$.

The disease-free equilibrium point $\left(E^{0}\right)$ is obtained when $I=0$, as follows

$$
E^{0}=(S, I, Q, R, B)=\left(\frac{\Lambda}{\mu}, 0,0,0,0\right) .
$$

Furthermore, when $I \neq 0$ we have the endemic equilibrium point $\left(E^{*}\right)$, that is 


$$
E^{*}=\left(S^{*}, I^{*}, Q^{*}, R^{*}, B^{*}\right)=\left(\frac{\Lambda-M I_{1}^{*}}{\mu}, I_{1}^{*}, \frac{\delta u I_{1}^{*}}{\varepsilon+\alpha_{2}+\mu}, \frac{\varepsilon \delta u I_{1}^{*}}{\mu\left(\varepsilon+\alpha_{2}+\mu\right)}, \frac{\mu M I_{1}^{*}}{\beta\left(\Lambda-M I_{1}^{*}\right)}\right),
$$

Where: $M=\delta u+\alpha_{1}+\mu$ and $I^{*}$ is the solution of polynomial of orde two

$$
\gamma K \beta^{2} M^{2} I^{2}-\left(\gamma K 2 \beta^{2} \Lambda M+b \mu^{2} M^{2}\right) I+b \mu M K \beta \Lambda-b \mu M^{2} \beta K+\gamma K \beta^{2} \Lambda^{2}=0 .
$$

The basic reproductive number $\left(R_{0}\right)$ in the epidemic model is unique and it determines the occurrence of an infectious disease (Heffernan et al., 2005). Furthermore, the next-generation matrix method (Brauer and Chavez, 2010) is used to determine the value of the basic reproduction number $\left(R_{0}\right)$, namely $R_{0}=\frac{\Lambda \gamma \beta}{\mu b(\delta u+\alpha+\mu)}$.

\section{Stability Analysis}

The model in (1.1) - (1.5) is a nonlinear system, therefore linearization is carried out around the equilibrium point to determine its stability. Based on the linearization process of model (1.1) - (1.5), the Jacobian matrix is given as follows.

$$
J=\left[\begin{array}{ccccc}
-\beta B-\mu & 0 & 0 & 0 & -\beta S \\
\beta B & -\left(\delta u+\alpha_{1}+\mu\right) & 0 & 0 & \beta S \\
0 & \delta u & -\left(\varepsilon+\alpha_{2}+\mu\right) & 0 & 0 \\
0 & 0 & \varepsilon & -\mu & 0 \\
0 & \gamma & 0 & 0 & b-\frac{2 b B}{K}
\end{array}\right]
$$

The stability of the disease-free equilibrium point $\left(E^{0}\right)$ is obtained by determining the eigenvalues of the Jacobian matrix system (1.1)-(1.5). $E^{0}$ is locally asymptotically stable when the real part of the eigenvalue is negative. However, based on the results, one of the eigenvalues is positive, which indicates an unstable disease-free equilibrium point $\left(E^{0}\right)$.

Similarly, the stability of $\mathrm{E}^{*}$ is obtained by determining the eigenvalues of the Jacobian matrix system. $E^{*}$ is locally asymptotically stable when it meets the criteria for the Routh Hurwitz [5]. Based on the results, the endemic point is asymptotically stable if only if

$$
\begin{aligned}
& a_{1}=\frac{(M+U+\mu) V+\beta \gamma W}{V}>0, \\
& a_{2}=\frac{(U+\mu) M V+\beta \gamma W(U+\mu)-\beta \gamma W U}{V}>0 .
\end{aligned}
$$

Where:

$U=\frac{\mu M I_{1}^{*}}{\Lambda-M I_{1}^{*}}$,

$V=b-\frac{2 b \mu M I_{1}^{*}}{K \beta\left(\Lambda-M I_{1}^{*}\right)}$,

$W=\frac{\Lambda-M I_{1}^{*}}{\mu}$.

\section{Numerical Simulation}

In this section, a numerical simulation is carried out using the Runge-Kutta order 4 method to describe the cholera epidemic model's dynamics with quarantine, using the parameter values in Table 1. 
The numerical simulations were carried out to illustrate the analyzes results previously described. Also, to show the instability of the disease-free equilibrium point, the parametric values were used in table 1 . Based on this parameter, the value $R_{o}=0.4311<1$. Similarly, one disease-free equilibrium point $E^{0}=\left(S^{0}, I^{0}, Q^{0}, R^{0}, B^{0}\right)=(0.1738,0,0,0,0)$ was obtained, while the endemic does not exist.

Table 1. Parameter values for numerical simulation

\begin{tabular}{|c|c|c|}
\hline Symbol & Parameter & Value \\
\hline$\Lambda$ & Natural birth rate, & $24.4 * 52 / 365000$ \\
\hline$\mu$ & Natural death rate, & 0.02 \\
\hline$\beta$ & The interaction rate of susceptible populations with bacteria, & 0.25 \\
\hline$\delta$ & Quarantine rate, & 0.05 \\
\hline$\alpha_{1}$ & Death rate due to infection, & 0.015 \\
\hline$\alpha_{2}$ & Death rate due to quarantine, & 0.0001 \\
\hline$\varepsilon$ & The individual rate is recovered, & 0.2 \\
\hline$K$ & Carrying capacity of bacteria population, & 10 \\
\hline$u$ & Control rate, & 0.1 \\
\hline$b$ & Bacteria population growth rate, & 0.001 \\
\hline$\gamma$ & The infected subpopulation triggers the bacteria growth rate. & 0.05 \\
\hline
\end{tabular}

Figure 1 shows that with initial values $(24,15,5,5,10)$, the solution does not lead to a disease-free $\left(E^{0}\right)$ or an endemic equilibrium point $\left(E^{*}\right)$. This is in line with the analysis results which states that $E^{0}$ is unstable.

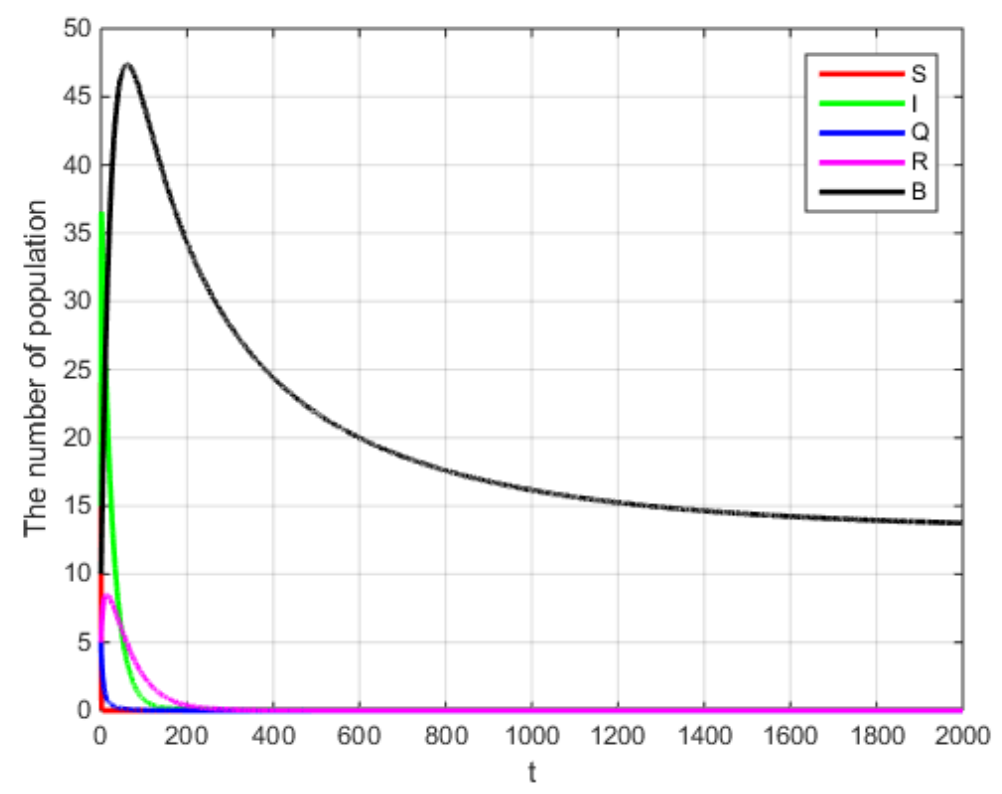

Figure 1. Model simulation for $R_{0}<1$

Furthermore, to illustrate the endemic equilibrium stability analysis results, the values in Table 1 are used with $\Lambda=0.2$. From this parameter, $R_{0}=24.8016>1$, one disease-free equilibrium point $E^{0}=\left(S^{0}, I^{0}, Q^{0}, R^{0}, B^{0}\right)=(10,0,0,0,0)$, and one endemic equilibrium point $E^{*}=(0.0145,4.9928$, 
$0.1134,1.1342,55.2134)$. Similarly, the value $a_{1}=0.1271>0$, and $a_{2}=0.1332>0$. The result indicates that the endemic equilibrium point is asymptotically stable. The simulation results also support this. Figure 2 shows that with the initial values $(24,15,5,5,10)$, the solution leads to the endemic equilibrium point $\left(E^{*}\right)$.

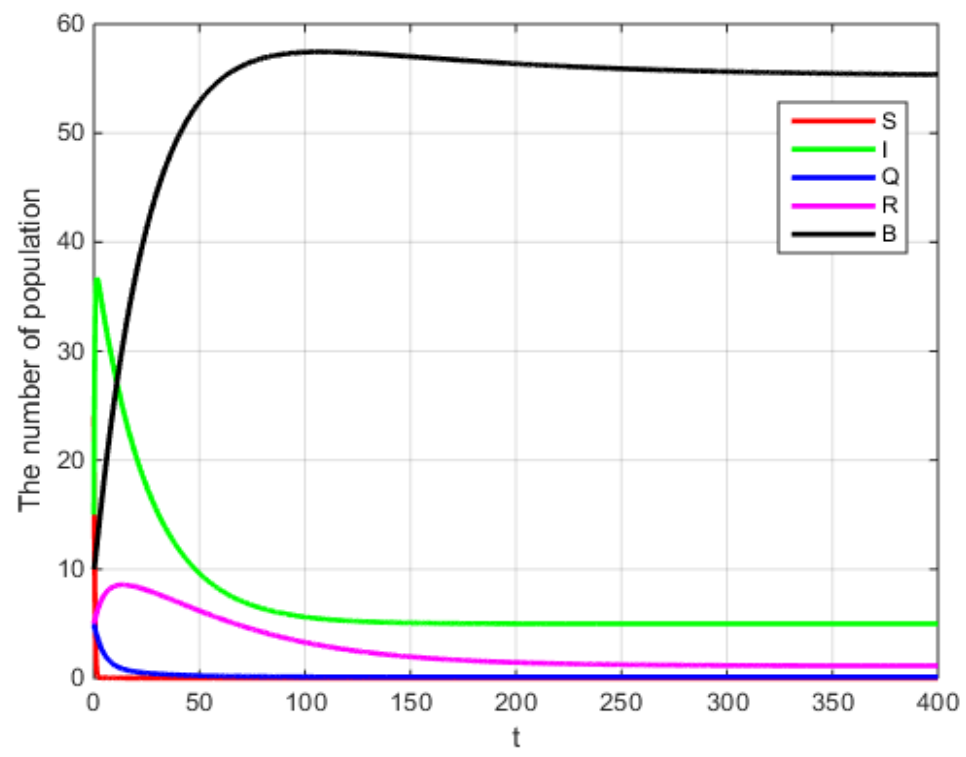

Figure 2. Model simulation for $R_{0}>1$

Figure 3 shows the model's simulation results with different quarantine rates, namely $\delta=0.01,0.05$, 0.1 and 0.5 . The higher the value, the less the spread of the infected subpopulation. This is consistent with the results that the basic reproduction rate $R_{0}$ is inversely proportional to $\delta$. Furthermore, the higher the quarantine rate, the lower the $R_{0}$ which results in a decrease in the spread of infection.

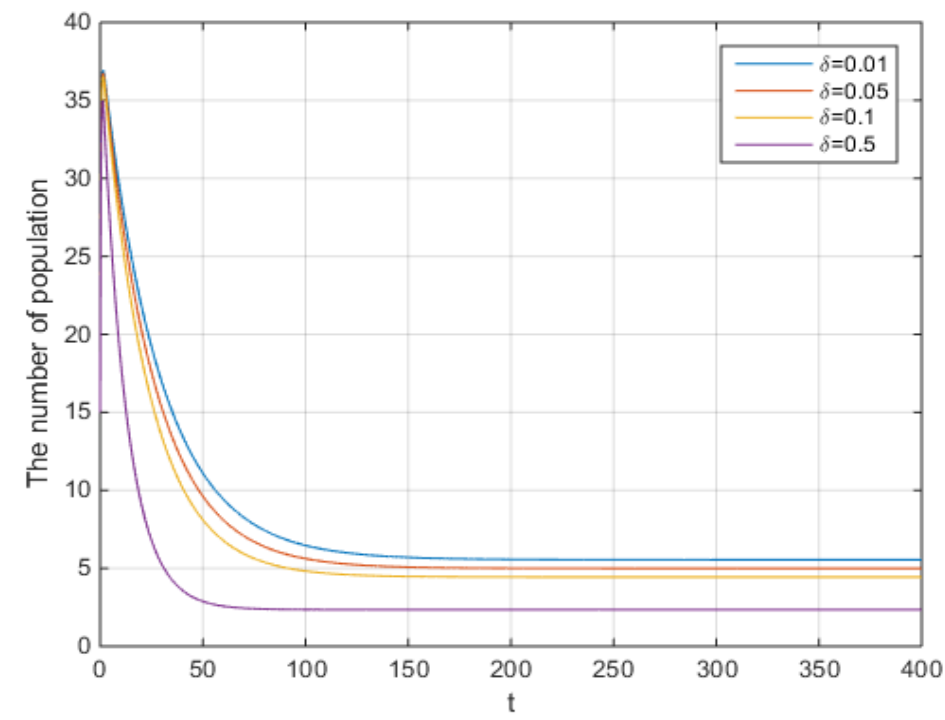

Figure 3. Model simulation with multiple quarantine rate values $(\delta)$. 


\section{CONCLUSION AND RECOMMENDATIONS}

The cholera model is a five-dimensional nonlinear autonomous system with 11 parameters and five dependent variables: $S, I, Q, R$, and $B$. In the $S I R Q B$ cholera model, two equilibrium points were obtained, namely the disease-free, which is always unstable and endemic, which is asymptotically stable under certain conditions. Furthermore, the numerical simulation of the local stability shows that the results are in line with the analysis. It also shows that the rate of quarantine has an impact on the spread of cholera infection. A higher rate implies a lower spread of cholera infection. Finally, further research is recommended to involve intervention with education and treatment or analyzed using optimal control.

\section{REFERENCES}

Berge, T., Bowong, S. \& Lubuma, J.M.S. (2015). Global Stability of a Two-Patch Cholera Model with Fast and Slow Transmissions. Mathematics and Computers in Simulation. 133: 142-164.

Berhe, H. W. (2020). Optimal Control Strategies and Cost_effectiveness Analysis Applied to Real Data of Cholera Outbreak in Ethiopia's Oromia Region. Chaos, Solitons and Fractals. 138

Brauer, F. \& Chavez, C. C. (2010). Mathematical Models in Population Biology and Epidemiology. Second Edition Springer Science+Business Media. New York.

Che, E.N., Kang, Y. \& Yakubu, Abdul-Aziiz. (2020). Risk Structured Model of Cholera Infections in Cameroon. Mathematical Biosciences. 320.

Codeco, C. T. (2001). Endemic and Epidemic Dynamics of Cholera: The Role of the Aquatic Reservoir. BMC Infectious Diseases. 1:1.

Edward S. \& Nyerere, N. (2015). A Mathematical Model for the Dynamics of Cholera with Control Measures. Applied and Computational Mathematics. 4: 53-63.

Fung, I. C. H., Fitter, D.L., Borse, R.H., Meltzer, M.I. and Tappero, J.W. (2013). Modeling the Effect of Water, Sanitation, and Hygiene and Oral Cholera Vaccine Implementation in Haiti. American Journal Tropical Medicine and Hygiene. 89(4): 633-640.

Hartley, D. M., Morris Jr, J.G. \& Smith, D.L. (2006). Hiperinfectivity: A Critical Element in the Ability of V. Cholerae to Cause Epidemics?. PLoS Medicine. 3(1): 63-68.

Heffernan, J. M., Smith, R. J., and Wahl, L. M. (2005). Perspectives on the Basic Reproductive Ratio. Journal of the Royal Society Interface. 2: 281-293.

Kolaye, G.G., Bowong, S., Houe, R., Aziz-Alaoui, M.A. \& Cadivel, M. (2018). Mathematical Assessment of the Role of Environmental Factors on the Dynamical Transmission of Cholera. Communications in Nonlinear Science and Numerical Simulation. 67: 203-222.

Lemos-Paiao, A. P., Silva, C.J. \& Torres, D.F.M. (2016). An Epidemic Model for Cholera with Optimal Control Treatment. Journal of Computational and Applied Mathematics. 318: 168-180.

Misra, A. K., Gupta, A. \& Venturino, E. (2016). Cholera Dynamics with Bacteriophage Infection a Mathematical Study. Nonlinear Science, Nonequilibrium and Complex Phenomena. 91: 610-621.

Panja, P. (2019). Optimal Control Analysis of a Cholera Epidemic Model. Biophysical Reviews and letters. 14: $1-22$.

Peter, O.J., Ayoade, A.A., Abioye, A. I., Victor, A.A. \& Akpan, C.E. (2018). Sensitivity Analysis of the Parameters of a Cholera Model. J. Applied Science Environ. Manage. 22(4): 477-481.

Sun, G. Q., Xie, J.H., Huang, S.H., Jin, Z. \& Li, M.T. (2016). Transmission Dynamics of Cholera Mathematical Modelling and Control Strategies. Communications in Nonlinear Science and Numerical Simulation. 45: 235-244. 
Tian, X., Xu, R. and Lin, J. (2019). Mathematical Analysis of a Cholera Infection Model with Vaccination Strategy. Applied Mathematics and Computation. 361: 517-535.

Zhou, X., Shi, X. \& Cui, J. (2016). Stability and Backward Bifurcation on a Cholera Epidemic Model with Saturated Recovery Rate. Mathematical Method in the Applied Sciences. 40: 1288-1306. 